\title{
Pressão intra-abdominal em cães ${ }^{1}$
}

\author{
Ronald Paiva M. Gonçalves ${ }^{2 *}$, Antonio Felipe P.F. Wouk ${ }^{3}$ e Anabella Mira ${ }^{4}$
}

\begin{abstract}
Gonçalves R.P.M., Wouk A.F.P.F. \& Mira A. 2011. [Intra-abdominal pressure in dogs.] Pressão intra-abdominal em cães. Pesquisa Veterinária Brasileira 31(9):812-816. Programa de Pós-Graduação em Farmacologia, Laboratório de Disfunções Cardiovasculares, Universidade Federal de Santa Catarina, Campus Universitário, Trindade, Bloco D/CCB, Florianópolis, SC 88049-900, Brazil. E-mail: ronaldmoreno.paiva@gmail.com

In order to favor the homogeneity of scientific studies and to provide guidelines on the diagnosis and therapy, human basal values of the intra-abdominal pressure (IAP) and their probable thresholds in morbid cases were recently established. However, basal values and changes in the intra-abdominal pressure in animals are still partly unknown and not scientifically confirmed. Veterinarians have the need to expand their knowledge on morbid conditions that develop with Intra-Abdominal Hypertension (IAH) or Abdominal Compartment Syndrome (ACS). The present study aims to test in dogs the technique adopted in human medicine as a model for measurement of intra-abdominal pressure (1) and determine normal values for the species (2). Fifteen known mongrel healthy dogs, males and females and homogenous were tested. The measurement of IAP was done through an indirect technique which involves catheterizing the bladder and using a water column with a graduate ruler in $\mathrm{cm}$ of $\mathrm{H}_{2} \mathrm{O}$ - its final value converted to $\mathrm{mm}$ of $\mathrm{Hg}$. The values observed ranged from a minimum sub atmospheric value (below of zero $\mathrm{mm}$ of $\mathrm{Hg}$ ) to $3,75 \mathrm{mmHg}$. There was a considerable variation within the individual values observed in some animals, bringing up considerations related to body mass, frequency/total volume of the physiologic solution to be administered and inquietude of these dogs during the treatment. The thresholds values found are considered physiologic, indicating reliability of the technique and possibility of its clinical use. The absence of sedation and the use of urethral catheter did not compromised the measurement, however could have resulted in under- or overestimation of the values found.
\end{abstract}

INDEX TERMS: Abdominal compartment syndrome, respiratory distress, cardiac insufficiency, oliguria, consensus.

RESUMO.- Sob consenso recente em pacientes humanos, os valores basais da pressão intra-abdominal (PIA) e seus prováveis limiares em casos mórbidos, foram estipulados com intuito de favorecer a homogeneidade de estudos científicos, além de fornecer diretrizes para conduta diagnóstica e tera-

\footnotetext{
${ }^{1}$ Recebido em 2 de fevereiro de 2011. Aceito para publicação em 25 de junho de 2011.

${ }^{2}$ Doutorando do Programa de Pós-Graduação em Farmacologia, Laboratório de Disfunções Cardiovasculares, Universidade Federal de Santa Catarina (UFSC), Campus Universitário, Trindade, Bloco D/CCB, Florianópolis, SC 88049-900, Brasil. *Autor para correspondência: ronaldmoreno.paiva@gmail.com

${ }^{3}$ Departamento de Medicina Veterinária, Universidade Federal do Paraná (UFPR), Rua dos Funcionários 1540, Bairro Cabral, Curitiba, PR 80035050, Brasil.

${ }^{4}$ Médica Veterinária Autônoma, Mestre em Ciências Veterinárias pela UFPR, Curitiba, PR. Endereço: Rua Terra Roxa 2491, Bairro Jardim La Salle, Toledo, PR 85903-020, Brasil.
}

pêutica destes pacientes. Valores basais e alterações na pressão intra-abdominal em animais não são ainda suficientemente conhecidos e cientificamente determinados. Médicos veterinários necessitam conhecer melhor os estados mórbidos que cursam com Hipertensão Intra-Abdominal (HIA) ou Síndrome de Compartimento Abdominal (SCA). 0 presente estudo objetivou testar em cães a técnica já descrita sob consenso na medicina como modelo de mensuração da pressão intra-abdominal, assim como determinar os valores normais para a espécie. Foram utilizados 15 cães hígidos, machos e fêmeas, homogêneos e todos sem raça definida. A mensuração da pressão intra-abdominal foi realizada por meio da técnica indireta de sondagem vesical e utilização de coluna de água com régua graduada em $\mathrm{cm}$ de $\mathrm{H}_{2} \mathrm{O}$, sendo seu valor final convertido para a unidade de $\mathrm{mm}$ de $\mathrm{Hg}$. Foi observado valor mínimo subatmosférico (abaixo de zero $\mathrm{mm}$ de $\mathrm{Hg}$ ) até o valor máximo de 3,75 mmHg. Houve importante variação 
entre os valores encontrados individualmente em determinados cães, colocando sob discussão questões como massa corporal, freqüência/volume total de solução fisiológica a ser infundida e agitação destes pacientes no momento do exame. Os valores limítrofes encontrados são considerados fisiológicos, indicando confiabilidade da técnica e possibilidade do seu emprego clínico. A ausência de sedação e utilização de sonda uretral não inviabilizou a realização da mensuração, porém podendo culminar numa sub ou superestimativa dos valores encontrados.

TERMOS DE INDEXAÇÃO: Síndrome de compartimento abdominal, disfunção respiratória, insuficiência cardíaca, oligúria, consenso.

\section{INTRODUÇÃO}

A cavidade abdominal é preenchida por órgãos caracterizados como relativamente não-compressíveis sendo delimitada por estruturas rígidas, tais como as vértebras espinhais, arco costal e pelve e por estruturas distensíveis, como a musculatura abdominal e diafragma, conferindo ao abdome certo grau de complacência não linear (em determinado momento um pequeno aumento do volume gera grandes elevações da pressão). Por conseqüência fisiológica, a cavidade abdominal apresenta determinada pressão descrita como Pressão Intra-Abdominal (PIA) (Malbrain et al. 2006).

A pressão intra-abdominal atua sinergicamente com eventos hemodinâmicos e com a ventilação/respiração, podendo sofrer modificações hiperagudas ou crônicas conseqüentes a alterações de volume da cavidade abdominal como a defecação (aguda) e ascite (crônica). A pressão intra-abdominal pode ser medida em $\mathrm{cm}$ de $\mathrm{H}_{2} \mathrm{O}$ ou $\mathrm{mm}$ de $\mathrm{Hg}$ por meio de técnica direta (inserção de cateter na cavidade abdominal ligado a um sistema de leitura) ou por técnica indireta via transdução da pressão de órgãos como a bexiga, estômago, útero ou cólon. Doentes que apresentem elevação sustentada da pressão intra-abdominal sofrem processo evolutivo para a denominada Hipertensão Intra-Abdominal (HIA) (Japiassú et al. 2007, Joubert et al. 2007).

Descrita pela primeira vez no homem na última metade do século 19, a HIA foi associada inicialmente a disfunções do sistema renal provocando quadros de oligúria ou mesmo anúria (Bradley \& Bradley 1947, Hunter \& Damani 2004). Efeitos deletérios na função cardíaca e pulmonar também foram descritos no século seguinte pela ação mecânica direta na cavidade abdominal, uma vez que esta culmina principalmente no deslocamento cranial do diafragma e compressão direta de plexos vasculares desde vênulas de parede fina, até grandes vasos como veia cava caudal e porta (Hunter \& Damani 2004, Ball et al. 2008).

0 deslocamento cranial do diafragma resulta na diminuição da complacência pulmonar por elevação da pressão intratorácica acarretando em aumento do trabalho ventilatório, "shunts" pulmonares e redução da capacidade residual funcional, representados por quadros de hipóxia e hipercapnia refratários à oxigenoterapia. Disfunções hemodinâmicas incoercíveis com diminuição do débito cardíaco são observadas em virtude da estenose/oclusão de vasos abdominais reduzindo a pré-carga e aumentando a póscarga de todo o leito arterial esplâncnico. A deterioração re- nal é creditada à compressão de seu plexo vascular associado à compressão do próprio parênquima, bem como pela resposta neuro-humoral decorrente da hipovolemia em analogia a um choque obstrutivo (Tiwari et al. 2002, Prado et al. 2005, Joubert et al. 2007).

A partir das disfunções orgânicas geradas principalmente no sistema respiratório, cardíaco e renal pela ação primária do aumento sustentado da PIA, introduziu-se em 1984, o termo Síndrome de Compartimento Abdominal (SCA). Atualmente esta entidade clínica é dividida em primária ou abdominal, secundária ou extra-abdominal e terciária ou recorrente, segundo consenso médico realizado em 2004 (Beck et al. 2004, Malbrain et al. 2006).

A SCA abdominal é associada com injúrias ou enfermidades da região abdomino-pélvica, tendo como exemplo os pacientes com traumatismo abdominal, hemoperitôneo, pancreatite aguda e os submetidos à intervenção hemostática emergencial com colocação de compressas no abdome. A SCA extra-abdominal origina-se de causas não primárias de acometimento do abdome, como, por exemplo, o paciente séptico, porém sendo seu maior exemplo, pacientes chocados submetidos à agressiva fluidoterapia ressuscitativa com conseqüente edema esplâncnico, da parede abdominal e retroperitoneal. Pacientes que apresentem agudização ou recorrência de uma SCA posteriormente ao tratamento médico ou cirúrgico são inseridos no conceito de SCA terciária (Malbrain et al. 2006, Ball et al. 2008).

Associadas à enfermidade de base, a HIA/SCA são consideradas fatores independentes de mortalidade em pacientes humanos, acrescido de comprovações, por estudos de prevalência em modelos críticos, de altas taxas de morbimortalidade devido à evolução para síndrome de disfunção de múltiplos órgãos (SDMO). Seu diagnóstico normalmente é demorado por se tratar de uma entidade de repercussão fisiopatológica multifatorial e insidiosa, concomitante ainda à falta de uniformidade das condutas em centros de terapia intensiva (Hong et al. 2002, Japiassú et al. 2007).

Originalmente descrito por Kron et al. (1984), o diagnóstico de elevação da PIA atualmente considerado como padrão ouro é baseado na técnica indireta de sondagem vesical, uma vez que a bexiga preenchida com pequenos volumes serve como reservatório passivo da PIA, permitindo sua mensuração sem qualquer influência da mesma. Inicialmente foi utilizada a infusão intravesical de $100 \mathrm{~mL}$ de solução salina $0,9 \%$ para mensuração da PIA em pacientes humanos, porém recentes trabalhos, acrescido do postulado consensual, indicam um volume de $25 \mathrm{~mL}$ da mesma solução, com padronização do posicionamento corporal, do nível que deve ser considerado o ponto zero do transdutor e o momento adequado de leitura para uma mensuração acurada da pressão intra-abdominal (Malbrain \& Deeren 2006).

0 exame clínico e a mensuração da circunferência abdominal de pacientes humanos com HIA/SCA não permitiram avaliar com acurácia o aumento da pressão intra-abdominal, sendo relatado como achados clínicos a ocorrência de abdome tenso, oligúria com hidratação normal e hipóxia refratária associada ao aumento de pressão da via aérea. A laparotomia descompressiva é considerada o único tratamento definitivo, sendo atualmente defendida também a utiliza- 
ção de estratégias medicamentosas na prevenção das disfunções ou falências de órgãos, com utilização principalmente de diuréticos e colóides (Sugrue et al. 2002, Tiwari et al. 2002, Cheatham et al. 2007).

A despeito das informações acerca da pressão intra-abdominal na medicina, a padronização da técnica para sua mensuração com conseqüente determinação de seus valores basais na espécie canina, bem como a incidência e prevalência de pacientes com HIA/SCA é ainda especulativa, em virtude da atual carência de publicações científicas sobre estas questões. 0 presente estudo teve por objetivos, mediante a mensuração da PIA em cães saudáveis pela técnica de sondagem intravesical, averiguar a aplicabilidade e reprodutibilidade do método e determinar os valores basais da PIA para a espécie canina.

\section{MATERIAL E MÉTODOS}

Foram utilizados 15 cães, machos e fêmeas, homogêneos e todos sem raça definida, atendidos no Serviço de Clínica Cirúrgica do Hospital Veterinário da UFPR - Curitiba. Como critério de inclusão apenas os animais considerados saudáveis, mediante avaliação clínica pelos mesmos profissionais, foram utilizados no experimento. Para as variáveis peso e idade são apresentadas as estatísticas descritivas de média e desvio padrão amostrais. 0 experimento foi previamente aprovado pela Comissão de Ética no Uso de Animais do Setor de Ciências Agrárias (CEUA SCA) da Universidade Federal do Paraná sob o protocolo de número 045/2008.

Os procedimentos de mensuração da pressão intra-abdominal seguiram a técnica sob consenso em medicina, sendo realizada a técnica indireta de sondagem vesical com utilização de sonda uretral adequada ao tamanho de cada paciente, uma torneira de três vias, equipo de pressão venosa central e régua graduada em $\mathrm{cm}$ de $\mathrm{H}_{2} \mathrm{O}$. Foi realizado o esvaziamento da bexiga seguido pela infusão de $25 \mathrm{~mL}$ de solução salina estéril a 0,9\%, subdividida em 5 infusões de $5 \mathrm{~mL}\left(\mathrm{I}_{1}-\mathrm{I}_{5}\right)$ com intervalo mínimo de 60 segundos para aferição do resultado, evitando possível ação do músculo detrusor da bexiga, e até que houvesse estabilização do menisco da coluna de água.

Os pacientes foram mantidos em decúbito lateral no decorrer do experimento, sendo a linha média pélvica utilizada como o ponto zero da coluna de água. Como preconizado pelo consenso médico, a título de padronização de resultados, os valores finais observados em $\mathrm{cm}$ de $\mathrm{H}_{2} \mathrm{O}$ sofreram conversão para a unidade de $\mathrm{mm}$ de $\mathrm{Hg}$ $\left(1,36 \mathrm{cmH}_{2} \mathrm{O}=1 \mathrm{mmHg}\right)$.

\section{RESULTADOS}

Entre os 15 cães estudados $\left(C_{1}-C_{15}\right)$ o peso variou de 17 a 29 $\mathrm{kg}$, com uma média de $22,46 \pm 2,85 \mathrm{~kg}$, enquanto o paciente mais jovem apresentava 1 ano e 6 meses e o mais velho 8 anos, estabelecendo média de 3,83 $\pm 1,55$ anos. Na amostra selecionada, $53,34 \%$ correspondem a machos e $46,66 \%$ a fêmeas.

Pela técnica indireta de sondagem vesical para mensuração da pressão intra-abdominal, foi observado valor mínimo subatmosférico (abaixo de zero $\mathrm{mm}$ de $\mathrm{Hg}$ ) até o valor máximo de $3,75 \mathrm{mmHg}$. Houve, em determinados cães, importante variação individual entre os valores encontrados, assim como em seus valores máximo e mínimo de pressão intra-abdominal nos diferentes momentos de aferição (Quadro 1).

0 valor máximo de pressão intra-abdominal observado $\left(\mathrm{C}_{11} \mathrm{I}_{4}\right)$ corresponde ao cão de maior idade na amostra (8
Quadro 1. Resultados das aferições seriadas da Pressão Intra-Abdominal (PIA) em quinze cães sabidamente hígidos pela técnica de sondagem vesical

\begin{tabular}{cccccc}
\hline & $\mathrm{I}_{1}$ (mmHg) & $\mathrm{I}_{2}$ (mmHg) & $\mathrm{I}_{3}(\mathrm{mmHg})$ & $\mathrm{I}_{4}(\mathrm{mmHg})$ & $\mathrm{I}_{5}(\mathrm{mmHg})$ \\
\hline $\mathrm{C}_{1}$ & Subatm $^{\mathrm{a}}$. & Subatm. & 2,43 & Subatm. & 0,22 \\
$\mathrm{C}_{2}$ & Subatm. & Subatm. & Subatm. & 2,65 & 2,35 \\
$\mathrm{C}_{3}$ & Subatm. & Subatm. & Subatm. & 0,29 & 0,15 \\
$\mathrm{C}_{4}$ & Subatm. & 1,40 & 2,43 & 2,21 & 2,13 \\
$\mathrm{C}_{5}$ & Subatm. & 0,59 & Subatm. & Subatm. & Subatm. \\
$\mathrm{C}_{6}$ & 0,51 & 0,51 & 0,96 & 1,84 & 1,77 \\
$\mathrm{C}_{7}$ & Subatm. & Subatm. & 0,59 & 0,96 & 0,44 \\
$\mathrm{C}_{8}$ & Subatm. & 0 & 1,25 & Subatm. & Subatm. \\
$\mathrm{C}_{9}$ & 0,88 & 0,51 & 0,22 & 0,74 & 1,10 \\
$\mathrm{C}_{10}$ & Subatm. & Subatm. & Subatm. & Subatm. & Subatm. \\
$\mathrm{C}_{11}$ & 1,10 & 2,06 & 3,02 & 3,75 & 3,16 \\
$\mathrm{C}_{12}$ & 2,50 & 0,22 & 1,03 & 0,96 & 1,47 \\
$\mathrm{C}_{13}$ & Subatm. & Subatm. & Subatm. & Subatm. & Subatm. \\
$\mathrm{C}_{14}$ & Subatm. & Subatm. & Subatm. & 0,37 & 1,69 \\
$\mathrm{C}_{15}$ & 0,22 & Subatm. & 0,37 & 1,54 & 1,54 \\
\hline
\end{tabular}

$\overline{\text { a Subatmosférico. }}$

anos), porém não sendo este o com a maior massa corporal (18 kg).

\section{DISCUSSÃO}

A partir da primeira descrição sobre pressão intra-abdominal, inúmeros estudos foram realizados em medicina com o intuito de padronizar a técnica de aferição e estabelecer quais seriam os valores limítrofes. Diferentes modelos experimentais foram avaliados com o intuito de caracterizar o risco de desenvolvimento de hipertensão intra-abdominal e/ou síndrome de compartimento abdominal (Biancofiore et al. 2002, Hong et al. 2002, Chen et al. 2008). Em 2004 no Congresso Mundial em Síndrome de Compartimento Abdominal (World Congress on Abdominal Compartment Syndrome, WSACS), foi estabelecido, por meio de definições consensuais, o valor mínimo subatmosférico até o valor máximo de $15 \mathrm{mmHg}$, sendo o último observado em pessoas com obesidade mórbida e mulheres grávidas (Malbrain et al. 2006, Cheatham et al. 2007).

Atualmente é preconizada como modelo ideal a técnica indireta de transdução vesical com infusão de pequenos volumes $(25 \mathrm{~mL})$ de solução fisiológica estéril, realização da leitura da pressão intra-abdominal (expressa em mm de $\mathrm{Hg}$ ) após 30 a 60 segundos para que não haja interferência de contração do músculo detrusor da bexiga, o decúbito supino, a colocação do transdutor na linha média da pelve e a averiguação da ausência de contração da musculatura abdominal, condutas estas que evitam superestimar a pressão intra-abdominal (Malbrain et al. 2008).

Em momentos anteriores à realização do consenso em medicina, havia muitas dúvidas quanto à realização da técnica de transdução vesical, principalmente quanto ao volume total a ser infundido no interior da bexiga, o tempo mínimo que se deve transcorrer da infusão para a aferição da pressão intra-abdominal sem a ação do músculo detrusor da bexiga, o posicionamento do paciente e o momento respiratório (se inspiratório ou expiratório) no ato da aferição (Kron et al. 1984, Malbrain \& Deeren 2006, Malbrain et al. 2008).

Publicações científicas acerca da pressão intra-abdominal em cães, sua aferição e pacientes que tenham risco para 
a hipertensão intra-abdominal ou síndrome de compartimento abdominal são escassas, e basearam-se quase todas em estudos médicos (Joubert et al. 2007). De maneira similar aos trabalhos em humanos, pesquisas em cães foram delineadas sem padronização de técnica, valendo-se da literatura humana ainda sem a análise consensual.

Em trabalho publicado recentemente por Joubert et al. (2007), foi observado aumento de pressão intra-abdominal em uma cadela de 9 anos da raça Bull Terrier com babesiose complicada (enfermidade per se acrescida da disfunção ou falência de um ou mais órgãos). Foram infundidos $50 \mathrm{~mL}$ de solução fisiológica estéril após completo esvaziamento da bexiga e leitura em coluna de água, tendo sido fixado o ponto zero na altura da linha média pélvica com o animal em decúbito lateral. Foram realizadas três mensurações no decorrer de 30 minutos, encontrando-se valores entre 17,63 e $18,75 \mathrm{mmHg}$, porém não informando qual sonda foi utilizada para a cateterização vesical, bem como o tempo de intervalo entre as mensurações. 0 diagnóstico de babesiose complicada foi baseado pelo surgimento de oligúria e azotemia a despeito da terapia adequada de reposição de volume. Além da disfunção renal, a HIA também contribuiu para a distensão abdominal, dispnéia, intolerância ao exercício e edema pulmonar, contribuindo com a deterioração clínica e o tratamento laborioso do animal.

Drellich (2000), em revisão acerca da PIA e SCA, reportou como valores fisiológicos de pressão intra-abdominal de cães o intervalo de 0 a $3,75 \mathrm{mmHg}\left(0\right.$ a $\left.5 \mathrm{~cm} \mathrm{H}_{2} \mathrm{O}\right)$, corroborando os achados deste estudo; a autora também observou a ocorrência de alterações hemodinâmicas, disfunção cardíaca e deterioração clínica em decorrência de aumento na PIA (acima de $3,75 \mathrm{mmHg}$ ) após a realização de colecistectomia por peritonite biliar.

Sinais clínicos indicativos e alterações patofisiológicas da hipertensão intra-abdominal têm sido descritas em cães, bem como em cavalos com cólicas, porém sem comprovações científicas (Joubert et al. 2007). Para a mensuração da pressão intra-abdominal a técnica de transdução intravesical é considerada segura, de fácil aplicabilidade e de baixo custo em virtude do material utilizado. É preconizado o uso de sonda do tipo Foley (possui o sistema de "cuff") para a cateterização vesical, pois garante a presença das fenestrações no interior da bexiga e minimiza a possibilidade de perdas significativas da solução para a região uretral. Há necessidade, porém, da utilização de drogas anestésicas com intuito de relaxar os esfíncteres do trato urinário inferior. Segundo Malbrain \& Deeren (2006), a utilização de sedativos também possibilita menor agitação e contração da musculatura abdominal, aumentando a acurácia da mensuração da pressão intra-abdominal.

Com o intuito de minimizar os efeitos potencialmente deletérios e riscos inerentes à utilização de drogas anestésicas em animais com baixa reserva orgânica funcional em momento de uma SCA, acrescido a tentativa de facilitação de técnica, o presente estudo realizou a mensuração da PIA sem o emprego da anestesia e utilização de sondas uretrais do tipo Foley. A cateterização vesical foi realizada em ambiente ambulatorial com a utilização de sonda uretral para a mensuração da pressão intra-abdominal.
Apesar da observação de variações individuais nos resultados dos quinze cães estudados nos diferentes momentos de aferição (vide Quadro 1), a utilização da técnica pelos autores apresentou ampla aplicabilidade e confiabilidade de acordo com os valores da PIA encontrados, possibilitando o emprego da técnica. Entre os pontos cruciais potencialmente favorecedores para as variações individuais nos resultados da PIA que devem ser comprovados por mais estudos, ressalta-se a massa corporal, ausência de sedação, utilização de sonda uretral e principalmente o volume total a ser infundido no interior da bexiga e o tempo necessário para a ausência de contração do músculo detrusor. Os achados subatmosféricos, apesar de considerados fisiológicos, podem indicar a ocorrência de posicionamento inadequado do nível zero da régua de coluna d'água.

A utilização do postulado médico idealizado no WSACS permitiu reprodutibilidade da mensuração da pressão intraabdominal em cães, encontrando valores considerados fisiológicos para a espécie segundo Drellich (2000). Apesar de o modelo canino apresentar similaridades ao homem na evolução fisiopatológica das alterações da pressão intra-abdominal (HIA/SCA) se faz necessário a determinação espécie-específica dos valores considerados normais para a espécie, permitindo posteriormente avaliar quais seriam os critérios e limiares precípuos de inclusão do cão para o diagnóstico de hipertensão intra-abdominal e síndrome de compartimento abdominal (Villaça \& Mantovani 2006, Joubert et al. 2007).

Valores sustentados ou repetidos da pressão intra-abdominal iguais ou acima de $12 \mathrm{mmHg}\left(8,82 \mathrm{cmH}_{2} \mathrm{O}\right)$, concomitante à observação de fatores de risco presentes, embasam o diagnóstico de hipertensão intra-abdominal em seres humanos. Em casos de comprovação clínico/laboratorial de novas disfunções ou falências orgânicas em pacientes com valores de PIA iguais ou acima de $20 \mathrm{mmHg}\left(14,70 \mathrm{cmH}_{2} \mathrm{O}\right)$, tem-se o diagnóstico de síndrome de compartimento abdominal (Malbrain et al. 2008).

As escassas publicações de revisão acerca da HIA/SCA e relatos de caso impossibilitam até o momento um postulado na determinação espécie-específica da HIA e SCA em pequenos animais. Segundo Drellich (2000), valores de PIA acima de $5 \mathrm{cmH}_{2} \mathrm{O}$ embasam o diagnóstico de HIA, enquanto a SCA desenvolve-se em ocasiões de PIA acima de $25 \mathrm{cmH}_{2} \mathrm{O}$, favorecendo o surgimento de disfunções orgânicas. Independentemente do acervo de publicações, é clara a correlação entre reduções da HIA e melhora clínica de pacientes veterinários e humanos, tornando o emprego da mensuração da PIA um parâmetro valioso na decisão de condutas terapêuticas adicionais e consequente redução da morbimortalidade.

É crescente a caracterização dos inúmeros fatores de risco que incluem pacientes humanos e caninos para o desenvolvimento de hipertensão intra-abdominal e síndrome de compartimento abdominal (Hong et al. 2002, Joubert et al. 2007, Ball et al. 2008); cirurgias abdominais extensas, traumatismo, reposição volêmica excessiva com edema esplâncnico, pancreatite, presença de massas e/ou efusões abdominais, síndrome de resposta inflamatória sistêmica (SRIS) e sepse são possíveis condicionantes para o seu surgimento (Peng et al. 2008). Considerando o caráter insidioso da HIA, a avaliação precoce da PIA torna-se, por con- 
seguinte, metodologia a ser empregada na rotina da clínica de pequenos animais.

O desenvolvimento, porém, de novas pesquisas multicêntricas para a determinação de valores fisiológicos e patológicos em cães, a partir do estabelecimento de padronização da técnica de mensuração da pressão intra-abdominal, será de grande relevância para o melhor entendimento desta entidade clínica. 0 posterior confronto de técnicas e resultados poderá convergir para um consenso, o que possibilitará abordagens diagnósticas e terapêuticas padronizadas pelos próprios médicos veterinários, auxiliando o diagnóstico precoce de pacientes com hipertensão intra-abdominal.

\section{CONCLUSÕES}

A utilização da técnica indireta de aferição da PIA pelo método de transdução intravesical demonstrou ser um procedimento de baixo custo, fácil aplicabilidade, além de segura para o paciente, sendo passível de reprodutibilidade.

Os limiares máximo e mínimo da pressão intra-abdominal encontrados nos 15 cães estudados são considerados fisiológicos, indicando a confiabilidade da técnica empregada, bem como sua aplicabilidade nesta espécie.

\section{REFERÊNCIAS}

Ball C.G., Kirkpatrick A.W. \& McBeth P. 2008. The secondary abdominal compartment syndrome: Not just another post-traumatic complication. Can. J. Surg. 51:399-405.

Biancofiore G., Bindi L., Romanelli A.M., Bisà M., Boldrini A., Consani G., Danella A., Urbani L., Filliponi F. \& Mosca F. 2002. Renal failure and abdominal hypertension after liver transplantation: Determination of critical intra-abdominal pressure. Liver Transplantation 8:1175-1181.

Bradley S.E. \& Bradley G.P. 1947. The effect of increased intra-abdominal pressure on renal function in man. J. Clin. Invest. 26:1010-1022.

Cheatham M.L., Malbrain M.L.N.G., Kirkpatrick A., Sugrue M., Parr M., Waele J., Balogh Z., Leppäniemi A., Olvera C., Ivatury R., D’Amours S., Wendon J., Hillman K. \& Wilmer A. 2007. Results from the international conference of experts on intra-abdominal hypertension and abdominal compartment syndrome. II. Recommendations. Intensive Care Medicine 33:951-962.

Chen H., Li F., Sun J. \& Jia J. 2008. Abdominal compartment syndrome in patients with severe acute pancreatitis in early stage. World J. Gastroenterol. 14(22):3541-3548.
Drellich S. 2000. Intra-abdominal and abdominal compartment syndrome. Comp. Cont. Educ. Pract. Vet. 22(8):764-768.

Hong J.J., Cohn S.M., Perez J.M., Dolich M.O., Brown M. \& McKenney M.G. 2002. Prospective study of the incidence and outcome of intra-abdominal hypertension and the abdominal compartment syndrome. Brit. J. Surgery 89:591-596.

Hunter J.D. \& Damani Z. 2004. Intra-abdominal hypertension and the abdominal compartment syndrome. Anaesthesia 59:899-907.

Japiassú A.M., Falcão H., Freitas F., Freitas S., Souza P.C.P., Lannes R., Sato R.I., Dias A.M., Almeida G.F., Soares M. \& Salluh J.I. 2007. Mensuração da pressão intra-abdominal nas unidades de tratamento intensivo: a opinião dos médicos intensivistas. Revta Bras. Terapia Intensiva 19:2:186-191.

Joubert K.E., Oglesby P.A., Downie J. \& Serfontein T. 2007. Abdominal compartment syndrome in a dog with babesiosis. J. Vet. Emerg. Critical Care 17(2):184-190.

Kron I.L., Harman P.K. \& Nolan S.P. 1984. The measurement of intra-abdominal pressure as a criterion for abdominal re-exploration. Ann. Surg. 199:28-30.

Malbrain M.L.N.G., Cheatham M.L., Kirkpatrick A., Sugrue M., Parr M., Waele J., Balogh Z., Leppäniemi A., Olvera C., Ivatury R., D'Amours S., Wendon J., Hillman K., Johansson K., Kolkman K. \& Wilmer A. 2006. Results from the international conference of experts on intra-abdominal hypertension and abdominal compartment syndrome. I. Definitions. Intensive Care Medicine 32:1722-1732.

Malbrain M.L.N.G. \& Deeren D.H. 2006. Effect of bladder volume on measured intravesical pressure: A prospective cohort study. Critical Care 10:R98.

Malbrain M.L.N.G., Vidts W., Ravyts M., Laet I. \& Waele J. 2008. Acute intestinal distress syndrome: the importance of intra-abdominal pressure. Minerva Anestesiologica 74:657-673.

Peng Z.Y., Critchley L.A., Joynt G.M., Gruber P.C., Jenkins C.R. \& Ho A.M.-H. 2008. Effects of norepinephrine during intra-abdominal hypertension on renal blood flow in bacteremic dogs. Crit. Care Med. 36:834-841.

Prado L.F.A., Júnior A.A., Cardoso E.S., Andrade R.S., Andrade R.S. \& Fernandes M.K. 2005. Pressão intra-abdominal em pacientes com trauma abdominal. Revta Col. Bras. Cir. 32:83-89.

Sugrue M., Bauman A., Jones F., Bishop G., Flabouris A., Parr M., Stewart A., Hillman K. \& Deane S.A. 2002. Clinical examination is an inaccurate predictor of intraabdominal pressure. World J. Surg. 26:1428-1431.

Tiwari A., Haq A.I., Myint F. \& Hamilton G. 2002. Acute compartment syndromes. Brit. J. Surg. 89:397-412.

Villaça M.P. \& Mantovani M. 2006. Comportamento da pressão intracraniana, da perfusão cerebral e dos parâmetros hemodinâmicos durante a síndrome do compartimento abdominal em cães. Revta Col. Bras. Cirur. 33:211-214. 